\title{
ENDOMORPHISMS OF RELATIVELY HYPERBOLIC GROUPS
}

\author{
IGOR BELEGRADEK AND ANDRZEJ SZCZEPAŃSKI
}

WITH APPENDIX BY OLEG V. BELEGRADEK

\begin{abstract}
We generalize some results of Paulin and Rips-Sela on endomorphisms of hyperbolic groups to relatively hyperbolic groups, and in particular prove the following.

- If $G$ is a non-elementary relatively hyperbolic group with slender parabolic subgroups, and either $G$ is not co-Hopfian or $\operatorname{Out}(G)$ is infinite, then $G$ splits over a slender group.

- If $H$ is a non-parabolic subgroup of a relatively hyperbolic group, and if any isometric $H$-action on an $\mathbb{R}$-tree is trivial, then $H$ is Hopfian.

- If $G$ is a non-elementary relatively hyperbolic group whose peripheral subgroups are finitely generated, then $G$ has a non-elementary relatively hyperbolic quotient that is Hopfian.

- Any finitely presented group is isomorphic to a finite index subgroup of $\operatorname{Out}(H)$ for some group $H$ with Kazhdan property (T). (This sharpens a result of Ollivier-Wise).
\end{abstract}

\section{INTRODUCTION}

The Bestvina-Paulin method [Pau88, Pau91, Bes88], further developed by Sela, has been a key ingredient in much of recent work on endomorphisms of hyperbolic groups [Pau91, RS94, Sel95, Sel97, Sel99], on endomorphisms of geometrically finite Kleinian groups [Sel97, OP98, DP03], and in Sela's work on Tarski's problem [Sel02].

Very recently, Groves [Gro] generalized various results on endomorphisms of hyperbolic groups, notably the Sela's shortening argument, to relatively hyperbolic groups with finitely generated free abelian parabolic subgroups.

The point of this paper is to show that some of the easier applications of the Bestvina-Paulin method extend, with little effort, to relatively hyperbolic groups with fairly arbitrary parabolic subgroups. By "easier" we mean the results that do not require JSJ-decompositions or the shortening argument.

2000 Mathematics Subject classification. Primary 20F65. Keywords: relatively hyperbolic, automorphism group, Hopfian, co-Hopfian, property (T), splitting, actions on trees. 
Relatively hyperbolic groups were introduced by Gromov [Gro87], and in this paper we use the following version of Gromov's definition developed by Bowditch (see [Bow, Definition 1]).

Definition 1.1. Let $G$ be a group with a (possibly empty) family of subgroups $\mathcal{G}$. We say that $G$ is hyperbolic relative to $\mathcal{G}$ if $G$ acts properly discontinuously and isometrically on a proper, geodesic, hyperbolic metric space $X$ so that the induced $G$-action on the ideal boundary of $X$ is the action of a geometrically finite convergence group whose maximal parabolic subgroups are precisely the elements of $\mathcal{G}$, and whose limit set is the ideal boundary of $X$. Elements of $\mathcal{G}$ are called peripheral subgroups.

Other definitions of relatively hyperbolic groups were developed by Farb [Far98], Bowditch [Bow, Definition 2], Yaman [Yam04], Druţu-Osin-Sapir [DS05], Osin [Osi06b], Druţu [Dru], and Mineyev-Yaman [MY]. It is known that all these definitions are equivalent to Definition 1.1, provided

$G$ and all its peripheral subgroups are finitely generated and infinite,

in which case we say that $G$ satisfies condition $(E)$. The proofs of various equivalences can be found in [Dah03b, Appendix A] (cf. [Bow, Szc98, Bum05]), [Osi06b, Theorem 7.10], [Yam04], [DS05, Theorem 8.5], [Dru, Theorems 4.21,4.34], [MY, Theorem 57].

We take this opportunity to correct a slight inaccuracy in [Osi06b, Theorem 7.10] and [Bum05] where the condition (E) is stated without requiring that the peripheral subgroups are infinite. In fact, in Definition 1.1 each peripheral subgroup is necessarily infinite (except when $\mathcal{G}=\emptyset$ ) because parabolic subgroups of a convergence group are infinite. By contrast, the definitions in [Far98, Osi06b, DS05, Dru, MY] allow elements of $\mathcal{G}$ to be finite, and are equivalent without requiring that elements of $\mathcal{G}$ are infinite. It turns out that allowing finite peripheral subgroups does not enlarge the class of relatively hyperbolic groups, namely, Osin proved (for his definition) that if $G$ is hyperbolic relative to $\mathcal{G}$, then for any conjugacy invariant subcollection $\mathcal{F} \subset \mathcal{G}$ of finite subgroups, $G$ is hyperbolic relative to $\mathcal{G} \backslash \mathcal{F}$ [Osi06b, Theorem 2.40]; thus by making $\mathcal{G}$ smaller, one can arrange that either all peripheral subgroups are infinite, or else $\mathcal{G}=\emptyset$.

Note that the group $G$ in Definition 1.1 satisfies (E) if and only if each peripheral subgroup of $G$ is finitely generated. Indeed, in this case Definition 1.1 is equivalent to [Bow, Definition 2], which means in particular that $G$ acts simplicially on a connected graph such that all vertex stabilizers are finitely generated, and the quotient graph is finite, which easily implies that $G$ is finitely generated (in fact a much more general result is proved in [Bro87, Theorem 2.2]). 
Very recently Hruska announced that Definition 1.1 is equivalent to Osin's definition even when $G$ or its peripheral subgroups are not finitely generated. This would give many examples of infinitely generated groups $G$ satisfying Definition 1.1. However, the only results of this paper that do not need $G$ to be finitely generated are Theorem 1.2, and Corollaries 5.1, 6.1.

We refer to [Bow, Section 6], or [Bow99], or [Yam04, Section 5] for relevant background on convergence groups. A subgroup of a relatively hyperbolic group $G$ is called elementary if the limit set of its action as a convergence group on the Bowditch's boundary of $G$ contains at most two points, which happens exactly if the subgroup is finite, virtually- $\mathbb{Z}$, or parabolic. Otherwise, the subgroup is called non-elementary. The Tits Alternative for convergence groups [Tuk94] implies that any small subgroup of a relatively hyperbolic group is elementary, where a subgroup is called small if it contains no non-cyclic free subgroup.

Examples of relatively hyperbolic groups are:

- the free products of finitely many finitely generated groups are hyperbolic relative to the factors (because the free product action on the corresponding Bass-Serre tree satisfies the Bowditch's definition [Bow] of a relatively hyperbolic group).

- hyperbolic groups are hyperbolic relative to any conjugacy invariant collection $\mathcal{G}$ of quasi-convex subgroups such that any element of $\mathcal{G}$ is equal to its normalizer, and any two distinct elements of $\mathcal{G}$ have finite intersection [Bow, Theorem 7.11]. Any hyperbolic group is hyperbolic relative to the empty family of subgroups.

- geometrically finite isometry groups of Hadamard manifolds of negatively pinched sectional curvature as defined in [Bow95] are hyperbolic relative to the maximal parabolic subgroups. This includes complete finite volume manifolds of negatively pinched sectional curvature.

- Osin [Osi] developed small cancellation theory for relatively hyperbolic groups, and proved that small cancellation quotients of relatively hyperbolic groups are hyperbolic relative to the images of maximal parabolic subgroups. These methods imply [AMO] that any two non-elementary finitely generated relatively hyperbolic group have a common quotient that is non-elementary relatively hyperbolic; in particular, since there are hyperbolic groups with Kazhdan property $(\mathrm{T})$, one can arrange the common quotient to have property $(\mathrm{T})$.

- combinations theorems for relatively hyperbolic groups were proved in [Dah03a, Osi06a], e.g. the amalgamation of relatively hyperbolic groups over parabolic subgroups is relatively hyperbolic, when the parabolic subgroup is maximal in at least one of the factors. 
- Hruska-Kleiner [HK05] proved that CAT(0)-groups with isolated flats are hyperbolic relative to the flat stabilizers. Examples of CAT(0)groups with isolated flats are listed in [HK05].

- Sela's limit group are hyperbolic relative to non-cyclic maximal abelian subgroups. This was first proved in [Dah03a] using a combination theorem, and in fact according to [AB06] limit groups are CAT(0)-groups with isolated flats.

The main observation is as follows.

Theorem 1.2. Let $H$ be a finitely generated group and $G$ be a non-elementary relatively hyperbolic group.

(i) If $\rho_{k}: H \rightarrow G$ is an arbitrary sequence of pairwise $G$-non-conjugate homomorphisms whose images are not parabolic, then $H$ admits a nontrivial action on an $\mathbb{R}$-tree $T$.

(ii) Furthermore, if all $\rho_{k}$ are injective, and $H_{a}$ is an arbitrary arc stabilizer of the $H$-action on $T$, then each finitely generated subgroup of $H_{a}$ is isomorphic to an elementary subgroup of $G$.

\section{Proof of Theorem 1.2}

Proof. Let $(X, d)$ be a proper, geodesic, $\delta$-hyperbolic metric space from the definition of a relative hyperbolic group. Choose a fundamental domain $F$ for the $G$-action on $X$. Fix a finite generating set $S$ for $H$. For $x \in X$ we let

$$
\mu_{k}(x)=\max _{s \in S} d\left(x, \rho_{k}(s)(x)\right),
$$

and let $\mu_{k}:=\inf _{x \in X} \mu_{k}(x)$. Choose $x_{k} \in X$ with $\mu_{k}\left(x_{k}\right) \leq \mu_{k}+\frac{1}{k}$.

First we show that $\mu_{k}$ has no bounded subsequence. Arguing by contradiction, consider a bounded subsequence, which we still denote $\mu_{k}$. Let $R=1+$ $\sup _{k} \mu_{k}$. Choose a $G$-invariant system of horoballs in $X$ such that any two horoballs are at least $R$ apart. There exists a sequence $g_{k} \in G$ such that $g_{k}\left(x_{k}\right) \in F$. If the sequence $g_{k}\left(x_{k}\right)$ is precompact, then since $G$ acts properly discontinuously, $g_{k} \rho_{k} g_{k}^{-1}$ fall into finitely many $G$-conjugacy classes, which contradicts the assumption. If $g_{k}\left(x_{k}\right)$ is not precompact, then after passing to a subsequence $g_{k}\left(x_{k}\right)$ lie in a horoball $\mathcal{H}$, and since horoballs are at least $R$ apart, $g_{k} \rho_{k}(s) g_{k}^{-1}\left(g_{k}\left(x_{k}\right)\right) \in \mathcal{H}$ for each $s \in S$. Hence $g_{k} \rho_{k}(H) g_{k}^{-1}$ lies in the parabolic subgroup that stabilizes $\mathcal{H}$, which contradicts the assumption that $\rho_{k}(H)$ is not parabolic.

Then by the work of Bestvina-Paulin (see e.g. Sections 3.1-3.5 of [Bes02]), the pointed spaces $\left(\frac{1}{\mu_{k}} X, x_{k}\right)$ subconverges to an $\mathbb{R}$-tree $T$, and $\rho_{k}$ 's converge to a non-trivial isometric $H$-action on $T$, which proves (i). 
Assume $\rho_{k}$ are all injective. Let $a$ be an arc of $T$ with the stabilizer $H_{a}$ in $H$. Let $\check{H}_{a}$ be the subgroup of $H_{a}$ of index at most 2 that fixes $a$ pointwise.

Approximate $a$ by geodesic segments $a_{k}$ in $\frac{1}{\mu_{k}} X$, denote the midpoint of $a_{k}$ by $m_{k}$. Changing $\rho_{k}$ within its $G$-conjugacy class, we can assume $m_{k} \in F$.

By [Pau91, p. 341] (cf. [BS94, p. 284]), there exists a constant $C(\delta)$, such that for each $h, f \in \check{H}_{a}$ and all large $k$, we have $d\left(m_{k}, \rho_{k}([h, f])\left(m_{k}\right)\right)<C(\delta)$. It follows that for large enough $k$, the same inequality holds for any given finite collection of commutators in $\check{H}_{a}$.

First, we prove that if $m_{k}$ is precompact in $F$, then $H_{a}$ is small, hence elementary. Indeed, since $m_{k}$ is precompact, only finitely elements of $G$ can satisfy $d\left(m_{k}, g\left(m_{k}\right)\right)<C(\delta)$, because $G$ acts isometrically and properly discontinuously. We denote the number of such elements by $M$. If $H_{a}$ is not small, then $\check{H}_{a}$ contains a rank two free subgroup generated by $h_{1}, h_{2}$. By the previous paragraph, for all large enough $k, d\left(m_{k}, \rho_{k}\left(\left[h_{1}, h_{2}^{s}\right]\right)\left(m_{k}\right)<C(\delta)\right.$ for $s=1, \ldots, M+1$. So $\rho_{k}\left(\left[h_{1}, h_{2}^{s_{1}}\right]\right)=\rho_{k}\left(\left[h_{1}, h_{2}^{s_{2}}\right]\right)$ for some $s_{1} \neq s_{2}$. Hence $\rho_{k}\left(\left[h_{1}, h_{2}^{s_{1}-s_{2}}\right]\right)=1$, which is impossible since $\rho_{k}$ is injective and $h_{1}, h_{2}$ generate a free subgroup.

It remains to consider the case when the sequence $m_{k}$ is not precompact. Fix a $G$-invariant system of horoballs in $X$ such that any two horoballs are at least $C(\delta)+1$ apart. Passing to a subsequence we can assume that all $m_{k}$ lie in one horoball. Denote by $P$ the maximal parabolic subgroup of $G$ stabilizing this horoball. Since any two horoballs are at least $C(\delta)+1$ apart, and since elements of $G$ map horoballs to horoballs, the above mentioned result [Pau91, p. 341] implies that the $\rho_{k}$-image of any finite collection of commutators lies in $P$, provided $k$ is large enough.

Let $z \in \partial X$ be the unique fixed point of $P$. Note that $P$ is the stabilizer of $z$ in $G$. Let $\Gamma$ be an arbitrary finitely generated subgroup of $H_{a}$, and let $\check{\Gamma}:=\Gamma \cap \check{H}_{a}$; the index of $\check{\Gamma}$ in $\Gamma$ is $\leq 2$, so $\check{\Gamma}$ is a normal subgroup of $\Gamma$. If all commutators in $\check{\Gamma}$ have finite order (i.e. the commutator subgroup $[\check{\Gamma}, \check{\Gamma}]$ is torsion), then $\check{\Gamma}$ and $\Gamma$ must be small, hence $\rho_{k}(\Gamma)$ is elementary.

Suppose that there exists a commutator $q \in \check{\Gamma}$ of infinite order. Fix an arbitrary $s \in \check{\Gamma}$, and take $k$ sufficiently large so that the $\rho_{k}$-image of the commutators $s q s^{-1} q^{-1}$ and $q$ lies in $P$, hence $\rho_{k}\left(s q s^{-1}\right)$ lies in the intersection of $P$ and $\rho_{k}(s) P \rho_{k}\left(s^{-1}\right)$. Since each $\rho_{k}$ is injective, $\rho_{k}(q)$ has infinite order, hence the intersection of $P$ and $\rho_{k}(s) P \rho_{k}\left(s^{-1}\right)$ is infinite. Distinct maximal parabolic subgroups have finite intersection, so $P=\rho_{k}(s) P \rho_{k}\left(s^{-1}\right)$, i.e. $\rho_{k}(s) \in P$. Since $\check{\Gamma}$ is finitely generated, $\rho_{k}(\check{\Gamma}) \subset P$ if $k$ is large enough. To see that $\rho_{k}(\Gamma)$ lies in $P$ take an arbitrary $\gamma \in \rho_{k}(\Gamma)$ and note that the subgroup $\gamma \rho_{k}(\check{\Gamma}) \gamma^{-1}$ fixes both $\gamma(z)$ and $z$, because $\check{\Gamma} \unlhd \Gamma$. Since the group $\gamma \rho_{k}(\check{\Gamma}) \gamma^{-1}$ is infinite 
parabolic, it has a unique fixed point, so $\gamma(z)=z$. Then $\rho_{k}(\Gamma)$ lies in the stabilizer of $z$, which equals to $P$.

Remark 2.1. It follows from Theorem 1.2(ii) that if parabolic subgroups of $G$ are small, then each $H_{a}$ is small. The same is true if small is replaced by amenable, slender, finitely generated virtually nilpotent, finitely generated virtually abelian, etc. Also if parabolic subgroups of $G$ are free, and $G$ is torsion-free, then each $H_{a}$ is locally free.

\section{ENDOMORPHISMS AND ACTIONS ON $\mathbb{R}$-TREES}

Theorem 1.2 immediately implies the following corollary, which for hyperbolic groups is due to Paulin [Pau91].

Corollary 3.1. If a finitely generated non-elementary relatively hyperbolic group $G$ has infinite $\operatorname{Out}(G)$, then $G$ acts non-trivially on an $\mathbb{R}$-tree such that any finitely generated subgroup of each arc stabilizer is isomorphic to an elementary subgroup of $G$.

A group is called co-Hopfian if every injective endomorphism of the group is surjective.

Corollary 3.2. Suppose $G$ is a finitely generated non-elementary relatively hyperbolic group that contains no infinite torsion group and is not isomorphic to a parabolic subgroup of $G$. If $G$ is not co-Hopfian, then $G$ acts non-trivially on an $\mathbb{R}$-tree such that any finitely generated subgroup of each arc stabilizer is isomorphic to an elementary subgroup of $G$.

Proof. Arguing by contradiction, we wish to show that $G$ is co-Hopfian. In the proof of [RS94, Theorems 3.1] Rips-Sela establish the co-Hopf property for any group $G$ such that

(a) $G$ has no infinite torsion subgroup,

(b) up to conjugation there are only finitely many monomorphisms $G \rightarrow G$,

(c) the image of any monomorphism $r: G \rightarrow G$ has finite centralizer in $G$.

Now (a) is true by assumption, (b) follows from Theorem 1.2 and the contradiction assumption. To verify (c) note that $r(G)$ is non-elementary because we assumed $G$ is non-elementary, so in particular, it is not finite or virtually- $\mathbb{Z}$, and also by assumption $G$ is not isomorphic to a parabolic subgroup. As we note in the proof of Corollary 6.1, any non-elementary subgroup of a relatively hyperbolic group has finite centralizer.

Remark 3.3. Sela [Sel97] proved that a torsion-free hyperbolic group is coHopfian if and only if it is freely indecomposable. Delzant-Potyagailo characterized co-Hopfian geometrically finite Kleinian groups in terms of splittings [DP03]. 
Remark 3.4. We do not know whether the assumption in Corollary 3.2 " $G$ contains no infinite torsion group" can be dropped. What is actually used in the proof is that if $\phi$ is a non-surjective monomorphism of $G \rightarrow G$ and $A_{k, \phi}$ is the (necessarily finite) centralizer of the subgroup $\phi^{k}(G)$, then the torsion subgroup $A_{\phi}=\bigcup_{k} A_{k, \phi}$ is finite. ${ }^{1}$ The assumption " $G$ is not isomorphic to a parabolic subgroup of $G$ " in Corollary 3.2 holds e.g. if parabolic subgroups are small and $G$ is non-elementary, or if parabolic subgroups are co-Hopfian. This assumption cannot be dropped as is shown in the following example (recall that groups with property $(\mathrm{T})$ have no nontrivial actions on $\mathbb{R}$-trees).

Example 3.5. There exists a non-elementary torsion-free relatively hyperbolic group that is not co-Hopfian, and that has Kazhdan property (T).

Proof. Osin's small cancellation methods allow to construct relatively hyperbolic Kazhdan groups with prescribed maximal parabolic subgroups. Specifically, if $H$ be an infinite finitely generated group and $K$ is a torsion-free non-elementary hyperbolic Kazhdan group, then $G:=H * \mathbb{Z} * K$ is hyperbolic relative to the factors $H, \mathbb{Z}$. It follows from properties of free products that $K$ is a suitable subgroup of $G$ (see [Osi] for a definition). By [Osi, Theorem 2.4] there is an epimorphism $\eta: G \rightarrow \bar{G}$ such that $\eta(K)=\bar{G}$, the restriction of $\eta$ to $H \cup \mathbb{Z}$ is injective, $\bar{G}$ is (Osin) hyperbolic relative to $\eta(H), \eta(\mathbb{Z})$, and $\bar{G}$ is obtained from $G$ by adding finitely many relations. In particular, $\bar{G}$ is Kazhdan (because property (T) is inherited by quotients), $\bar{G}$ is non-elementary, and furthermore if $H$ is finitely presented, then so is $\bar{G}$. Moreover, by [Osi, Theorem 2.4] any element of finite order in $\bar{G}$ is the image of a finite order element in $G$, so if $H$ is torsion-free, then so are $G$ and $\bar{G}$. By construction, $G$ satisfies (E) so it is relatively hyperbolic in the sense of Definition 1.1. Now suppose $H$ is a universal torsion-free finitely presented group (see Theorem A.1 below), i.e. $H$ is a finitely presented torsion-free group such that any finitely presented torsion-free group embeds into $H$. Thus $\bar{G}$ embeds into $H \cong \eta(H) \subset \bar{G}$, hence $\bar{G}$ is not co-Hopfian.

Remark 3.6. Corollaries 3.1, 3.2 generally fail for finitely generated subgroups of hyperbolic groups: a non-co-Hopfian finitely generated subgroup of a hyperbolic group was constructed in [KW01], and by [OW] there exist Kazhdan groups that have infinite outer automorphism groups yet are embedded into hyperbolic group. However, the examples in [KW01, OW] are not finitely presented, and it seems no finitely presented examples are known.

\footnotetext{
${ }^{1}$ It was recently showed in [DS, Lemma 4.44] that $A_{\phi}$ is finite if $G$ satisfies (E).
} 


\section{SPlitTing OVER SLENDER GROUPS}

A group is called slender if all its subgroups are finitely generated. The class of slender group is closed under extensions [DS99], in particular, virtually polycyclic groups are slender. Slender groups are small because non-abelian free groups contain infinitely generated subgroups.

As explained in the introduction, if $G$ is a relatively hyperbolic group with slender (or more generally finitely generated) peripheral subgroups, then $G$ is finitely generated. Furthermore, by the Tits Alternative for relatively hyperbolic groups [Tuk94], small subgroups of $G$ are parabolic, hence slender. Then since in any group an ascending chain of small subgroups is small, every ascending chain of slender subgroups of $G$ stabilizes. Hence, any nontrivial minimal $G$-action on an $\mathbb{R}$-tree with slender arc stabilizers is stable [BF95], and therefore [BF95] if $G$ is finitely presented, then there exists a nontrivial $G$-action on a simplicial tree slender edge stabilizers, i.e. a splitting of $G$ over a slender subgroup. (Note that the splitting is nontrivial provided $G$ is non-elementary). In fact, according to Dunwoody [Dun, Theorem 2], the assumption " $G$ is finitely presented" can be replaced by " $G$ is finitely generated", which holds if $G$ is relatively hyperbolic with slender peripheral subgroups. Thus combining [Dun, Theorem 2] with Corollaries 3.1, 3.2, we get the results stated in the abstract about co-Hopf property and infinite outer automorphism group, as well as the following.

Corollary 4.1. Let $H$ be a finitely generated group that is not slender, and let $G$ be a relatively hyperbolic group with slender parabolic subgroups. If $\rho_{k}: H \rightarrow G$ is an arbitrary sequence of pairwise $G$-non-conjugate injective homomorphisms, then $H$ admits a nontrivial splitting over a slender group.

Remark 4.2. It is interesting to compare Corollary 4.1 with a (much more delicate) result of Dahmani [Dah06] who showed that if $H$ is a finitely presented group and $G$ is a relatively hyperbolic group, then up to $G$-conjugacy there are only finitely many subgroups of $G$ that are non-parabolic, do not split over parabolic subgroups, and are the images of homomorphisms $H \rightarrow G$.

\section{Subgroups With Property $F \mathbb{R}$ are Hopfian}

A group $H$ is said to have property $F \mathbb{R}$ if any $H$-action on an $\mathbb{R}$-tree fixes a point, i.e. is not non-trivial. Any group with property $F \mathbb{R}$ is finitely generated [Ser77, p.81]. By Theorem 1.2 if $H$ has property $F \mathbb{R}$, then there are only finitely many $G$-conjugacy classes of homomorphisms $H \rightarrow G$ with nonparabolic images.

The class of groups with property $F \mathbb{R}$ is closed under extensions [Ser77, p. 85] and quotients, and contains 
- groups with Kazhdan property (T) (this was proved in [Wat82] for simplicial trees, and extended to $\mathbb{R}$-trees in [Nos93]);

- $\operatorname{Aut}\left(F_{n}\right)$ and $\operatorname{Out}\left(F_{n}\right)$ for $n>2$ [CV96], where $F_{n}$ is a free group of rank $n$; mapping class groups $M_{g, r}$ for $g \geq 2$ [CV96];

- finitely generated Coxeter groups with no $\infty$ labels in their Coxeter diagrams (this is an exercise in [Ser77, p. 93]),

- fundamental groups of closed irreducible non-Haken 3-manifolds [MS88, Proposition 2.1].

Recall that group is called Hopfian if every surjective endomorphism of the group is injective.

Corollary 5.1. If a non-parabolic subgroup $H$ of a relatively hyperbolic group has property $F \mathbb{R}$, then $H$ is Hopfian.

Proof. If $\rho$ is a non-bijective epimorphism of $H$, then precomposing the inclusion $H \hookrightarrow G$ with the powers $\rho^{k}$, we get the homomorphisms $H \rightarrow G$ with image $H$, which is non-parabolic by assumption. To show that $\rho$ is injective, it is enough to prove that some power of $\rho$ is injective. Look at the sequence $\psi_{k}:=\rho^{2^{k}}$, so that for any two endomorphisms in the sequence $\left\{\psi_{k}\right\}$ the one with the larger index is a power of the other. By Theorem 1.2, after passing to a subsequence, we can assume that $\psi_{k}$ 's are conjugate in $G$. In particular, if $\psi$ denotes the first endomorphism in the subsequence, we get $\psi^{s_{k}}=i_{g_{k}} \circ \psi$, where $g_{k} \in G$ and $i_{g_{k}}$ is the corresponding inner automorphism of $G$. Then $\psi^{s_{k}}$ and $\psi$ have equal kernels. Take $\gamma \in \operatorname{ker}\left(\psi^{s_{k}-1}\right)$. Since $\psi$ is onto, we can find $\tilde{\gamma} \in H$ with $\psi(\tilde{\gamma})=\gamma$. So $\tilde{\gamma} \in \operatorname{ker}\left(\psi^{s_{k}}\right)=\operatorname{ker}(\psi)$, which implies $\gamma=1$, as wanted.

Corollary 5.2. If $G$ is non-elementary relatively hyperbolic group whose peripheral subgroups are finitely generated, then $G$ has a non-elementary relatively hyperbolic quotient $\bar{G}$ that is Hopfian. Also if $G$ is hyperbolic, then so is $\bar{G}$.

Proof. Since the peripheral subgroups are finitely generated, $G$ satisfies $(E)$, so Definition 1.1 is equivalent to Osin's definition for which it is known by [AMO] that any two finitely generated non-elementary relatively hyperbolic groups have a common non-elementary relatively hyperbolic quotient. Since there exists a non-elementary hyperbolic group with Kazhdan property (T), any nonelementary relatively hyperbolic group $G$ has a non-elementary (Osin) relatively hyperbolic quotient $\bar{G}$ with property $(\mathrm{T})$, which is Hopfian by Corollary 5.1. By [Osi, Theorem 2.4] (on which the results [AMO] are based) the surjection $G \rightarrow \bar{G}$ maps the peripheral subgroups of $G$ isomorphically onto the peripheral subgroups of $\bar{G}$, so $\bar{G}$ satisfies $(E)$ and hence $\bar{G}$ is also relatively hyperbolic in the sense of Definition 1.1. Finally, if $G$ is hyperbolic, we can take $\mathcal{G}$ to be empty, so that $\bar{G}$ has no peripheral subgroups, i.e. $\bar{G}$ is hyperbolic. 
Remark 5.3. That hyperbolic groups with property $F \mathbb{R}$ are Hopfian was noted to [RS94, Theorem 2.1], and Corollary 5.1 extends this result to relatively hyperbolic case. Later Sela [Sel99] showed that any torsion-free hyperbolic group is Hopfian, and more recently Bumagin proved [Bum04] that any finitely generated subgroup of a torsion-free hyperbolic group is Hopfian. Thus the assumption " $H$ has property $F \mathbb{R}$ " in Corollary 5.1 is probably far from optimal. On the other hand, there certainly exist non-Hopfian relatively hyperbolic groups, e.g. the free product of any two finitely generated groups one of which is nonHopfian.

Remark 5.4. Ollivier-Wise [OW] and de Cornulier [dC07] gave examples of non-Hopfian Kazhdan groups. By Corollary 5.1 these groups cannot be embedded in a relatively hyperbolic group as non-parabolic subgroups, because Kazhdan groups are finitely generated and have property $F \mathbb{R}$.

\section{Automorphism groups of KaZhdan groups}

Any hyperbolic group with Kazhdan property (T) has finite outer automorphism group [Pau91]. By contrast, Ollivier-Wise [OW] showed that any finitely presented group is isomorphic to the quotient of a torsion-free hyperbolic group $G$ by an infinite normal subgroup $H$ that has Kazhdan property (T). Then the canonical homomorphism $G / H \rightarrow \operatorname{Out}(H)$ is injective, thus Ollivier-Wise concluded that any finitely presented group embeds into $\operatorname{Out}(H)$ for some group $H$ with property (T). We note that the embedding $G / H \rightarrow \operatorname{Out}(H)$ necessarily has finite cokernel.

Corollary 6.1. If $H$ has property $F \mathbb{R}$ and is a normal subgroup of a nonelementary torsion-free relatively hyperbolic group $G$, then the canonical homomorphisms $G / H \cong \operatorname{Inn}(G) / \operatorname{Inn}(H) \rightarrow \operatorname{Out}(H)$ and $G \cong \operatorname{Inn}(G) \rightarrow \operatorname{Aut}(H)$ are embeddings onto finite index subgroups.

Proof. We think of $G$ as a discrete convergence group. Infinite elementary subgroups have elementary normalizers because the normalizer stabilizes the limit set of the subgroup. Since $H$ is normal and $G$ is non-elementary, we conclude that $H$ is non-elementary.

Now we show that non-elementary subgroups have finite centralizers. Indeed, if the centralizer $C_{G}(H)$ of $H$ in $G$ contains an infinite order element $c$, then $H$ fixes the limit set of the cyclic subgroup generated by $c$, so $H$ cannot be non-elementary. Thus $C_{G}(H)$ must be a torsion group, in particular, $C_{G}(H)$ is elementary, else $C_{G}(H)$ would have to contain a non-abelian free subgroup. If $C_{G}(H)$ is infinite, it has a nonempty limit set that must be fixed by $H$, contradicting the assumption that $H$ is non-elementary. 
Since $G$ is torsion free, $C_{G}(H)$ is trivial. Hence the canonical homomorphism $\operatorname{Inn}(G) \rightarrow \operatorname{Aut}(H)$ in injective, and we identify its image with $\operatorname{Inn}(G)$. By Theorem 1.2 applied to the inclusion $H \hookrightarrow G$ precomposed with automorphisms of $H, \operatorname{Inn}(G)$ has finite cokernel in $\operatorname{Aut}(H)$. Let $G_{0}$ be the intersection of all the conjugates of $\operatorname{Inn}(G)$ in $\operatorname{Aut}(H)$. Note that $G_{0}$ is a finite index normal subgroup of $\operatorname{Aut}(H)$ that contains $H$. The kernel of the surjection of $\operatorname{Out}(H)=$ $\operatorname{Aut}(H) / \operatorname{Inn}(H)$ onto the finite group $\operatorname{Aut}(H) / G_{0}$ equals to $G_{0} / \operatorname{Inn}(H) \cong$ $G_{0} / H$. Since $G_{0} / H \leq G / H \leq \operatorname{Out}(H)$, we conclude that $G / H$ has finite index in $\operatorname{Out}(H)$.

Corollary 6.2. Given a finitely presented group $Q$ there exists a group $H$ with Kazhdan property $(T)$ such that $Q$ is isomorphic to a finite index subgroup of $\operatorname{Out}(H)$, and the group $\operatorname{Aut}(H)$ is hyperbolic.

Proof. By $[\mathrm{OW}], Q \cong G / H$ where $G$ is hyperbolic and $H$ has property (T). By Corollary 6.1, $Q$ embeds into $\operatorname{Out}(H)$ as a finite index subgroup, and $\operatorname{Aut}(H)$ is hyperbolic because it contains the hyperbolic subgroup $G$ of finite index.

\section{Appendix A. On universal torsion-Free Finitely PRESENTED Groups,} By Oleg V. BelegradeK

Theorem A.1. There exists a universal torsion-free finitely presented group.

Proof. We will show below that there exists a torsion-free recursively presented group $P$ which contains an isomorphic copy of every torsion-free finitely presented group. (Note that this is not completely trivial: the naive idea "consider an effective listing of finite presentations of all torsion-free finitely presented groups and form the disjoint union of the presentations" fails because such a listing does not exist [Lem97].) By the Higman Embedding Theorem, $P$ embeds into a finitely presented group $H$ which is built from $P$ and the trivial group by a finite sequence of HNN-extensions and free products [Rot84, pp. 364-365, 389]. Since the class $\mathbb{T F}$ of all torsion-free groups is closed under HNN-extensions and free products, $H$ is torsion-free. Thus, $H$ is a universal torsion-free finitely presented group.

The class $\mathbb{T F}$ is a quasivariety specified by the recursive set $Q$ of quasi-identities $\forall x\left(x^{n}=1 \rightarrow x=1\right)$, where $n$ runs over positive integers. By general nonsense [Hod93, Theorem 9.2.2], any quasivariety admits presentations. We denote by $G^{\tau}$ the group defined in $\mathbb{T F}$ by a presentation $\tau$, and by $G_{\tau}$ the group defined by $\tau$ in the variety of all groups. Let $\left(\pi_{n}: n=0,1, \ldots\right)$ be an effective listing of all finite presentations. Clearly, the disjoint union $\pi$ of all $\pi_{n}$ is a recursively enumerable presentation. We show that one can take $G^{\pi}$ as $P$.

Any torsion-free finitely presented group embeds into $G^{\pi}$. Indeed, if $\tau$ is a finite presentation such that $G_{\tau}$ is torsion-free then $G_{\tau}=G^{\tau}$; clearly, $G^{\pi}$ 
contains an isomorphic copy of $G^{\tau}$. It remains to show that $G^{\pi}$ is recursively presented in the variety of all groups. Let $\pi=\langle X, R\rangle$, and $R^{\prime}$ be the set of all relations of $G^{\pi}$ in generators $X$. Then $w$ is in $R^{\prime}$ if and only if the formula $w=1$ is a consequence of the set of formulas $Q_{R}=Q \cup\{r=1: r \in R\}$ in first order logic [Hod93, Lemma 9.2.1]. Since $Q$ and $R$ are recursively enumerable, $Q_{R}$ is recursively enumerable, too. The set of consequences of any recursively enumerable set of axioms is recursively enumerable [Hod93, Lemma 6.1.3], hence $R^{\prime}$ is recursively enumerable, as claimed.

Remark A.2. The above argument applies verbatim to show that every quasivariety of groups $K$ defined by a recursively enumerable set of quasi-identities contains a group which is recursively presented in the variety of all groups and contains isomorphic copies of all finitely presented groups that belong to $K$. For the quasivariety $\mathbb{T F}$ there is a more constructive (but less elegant) proof that $G^{\pi}$ is recursively presented based on the following observation: $R^{\prime}=\bigcup_{n} R_{n}$, where $R_{0}$ is the normal closure of $R$ (in the free group generated by $X$ ), and $R_{n+1}$ is the normal closure of the set of all roots of all elements of $R_{n}$.

Acknowledgements: We are grateful to Oleg V. Belegradek, François Dahmani, Chris Hruska, Ilya Kapovich, Misha Kapovich, Denis Osin, Mark Sapir, and the referee for helpful communications. The first author was partially supported by the NSF grant \# DMS-0352576. The second author was partially supported by the Polish grant BW 5100-5-0096-5.

Added in April 2007: A version of this paper was posted as [BS] in January of 2005, and the only new results in the present version are Corollary 5.2 and Appendix A (whose sole purpose was to make the group in Example 3.5 torsionfree). Eleven months later Druţu-Sapir circulated a remarkable preprint [DS] in which they extended Rips theory to tree-graded spaces and, in particular, obtained generalizations of Theorem 1.2 and Corollaries 3.1, 3.2, 4.1. DruţuSapir's argument is different and quite long.

\section{REFERENCES}

[AB06] E. Alibegović and M. Bestvina, Limit groups are CAT(0), J. London Math. Soc. (2) 74 (2006), no. 1, 259-272.

[AMO] G. Arzhantseva, A. Minasyan, and D. Osin, The SQ-universality and residual properties of relatively hyperbolic groups, arXiv:math.GR/0601590.

[Bes88] M. Bestvina, Degenerations of the hyperbolic space, Duke Math. J. 56 (1988), no. 1, 143-161.

[Bes02], $\mathbb{R}$-trees in topology, geometry, and group theory, Handbook of geometric topology, North-Holland, Amsterdam, 2002, pp. 55-91.

[BF95] M. Bestvina and M. Feighn, Stable actions of groups on real trees, Invent. Math. 121 (1995), no. 2, 287-321.

[Bow] B. H. Bowditch, Relatively hyperbolic groups, Southampton preprint, 1999, www.maths.soton.ac.uk/staff/Bowditch/preprints.html. 
[Bow95] B. H. Bowditch, Geometrical finiteness with variable negative curvature, Duke Math. J. 77 (1995), no. 1, 229-274.

[Bow99] B. H. Bowditch, Convergence groups and configuration spaces, Geometric group theory down under (Canberra, 1996), de Gruyter, Berlin, 1999, pp. 23-54.

[Bro87] K. S. Brown, Finiteness properties of groups, J. Pure Appl. Algebra 44 (1987), no. 1-3, 45-75, Proceedings of the Northwestern conference on cohomology of groups (Evanston, Ill., 1985).

[BS] I. Belegradek and A. Szczepański, Endomorphisms of relatively hyperbolic groups, arXiv:math.GR/0501321.

[BS94] M. R. Bridson and G. A. Swarup, On Hausdorff-Gromov convergence and a theorem of Paulin, Enseign. Math. (2) 40 (1994), no. 3-4, 267-289.

[Bum04] I. Bumagin, Subgroups of torsion-free hyperbolic groups have the Hopf property, Geom. Dedicata 106 (2004), no. 1, 211-230.

[Bum05] - On definitions of relatively hyperbolic groups, Geometric methods in group theory, Contemp. Math., vol. 372, Amer. Math. Soc., Providence, RI, 2005, pp. 189 196.

[CV96] M. Culler and K. Vogtmann, A group-theoretic criterion for property FA, Proc. Amer. Math. Soc. 124 (1996), no. 3, 677-683.

[Dah03a] F. Dahmani, Combination of convergence groups, Geom. Topol. 7 (2003), 933-963 (electronic).

[Dah03b] _ Les groupes relativement hyperboliques et leurs bords, Ph.D. thesis, Université Louis Pasteur, Strasbourg, 2003.

[Dah06] - Accidental parabolics and relatively hyperbolic groups, Israel J. Math. 153 (2006), 93-127.

[dC07] Y. de Cornulier, Finitely presentable, non-Hopfian groups with Kazhdan's property (T) and infinite outer automorphism group, Proc. Amer. Math. Soc. 135 (2007), no. 4, 951-959.

[DP03] T. Delzant and L. Potyagailo, Endomorphisms of Kleinian groups, Geom. Funct. Anal. 13 (2003), no. 2, 396-436.

[Dru] C. Druţu, Relatively hyperbolic groups: geometry and quasi-isometric invariance, arXiv:math.GR/0605211.

[DS] C. Druţu and M. Sapir, Groups acting on tree-graded spaces and splittings of relatively hyperbolic group, arXiv:math.GR/0601305.

[DS99] M. J. Dunwoody and M. E. Sageev, JSJ-splittings for finitely presented groups over slender groups, Invent. Math. 135 (1999), no. 1, 25-44.

[DS05] C. Druţu and M. Sapir, Tree-graded spaces and asymptotic cones of groups, Topology 44 (2005), no. 5, 959-1058, With an appendix by D. Osin and Sapir.

[Dun] M. L. Dunwoody, Groups acting on real trees, Southampton preprint, 2004, www.maths.soton.ac.uk/staff/Dunwoody/Tess11.dvi.

[Far98] B. Farb, Relatively hyperbolic groups, Geom. Funct. Anal. 8 (1998), no. 5, 810-840.

[Gro] D. Groves, Limit groups for relatively hyperbolic groups, I: The basic tools, arXiv:math.GR/0412492.

[Gro87] M. Gromov, Hyperbolic groups, Essays in group theory, Math. Sci. Res. Inst. Publ., vol. 8, Springer, 1987, pp. 75-263.

[HK05] G. C. Hruska and B. Kleiner, Hadamard spaces with isolated flats, Geom. Topol. 9 (2005), 1501-1538 (electronic), With an appendix by the authors and M. Hindawi.

[Hod93] W. Hodges, Model theory, Cambridge University Press, 1993.

[KW01] I. Kapovich and D. T. Wise, On the failure of the co-Hopf property for subgroups of word-hyperbolic groups, Israel J. Math. 122 (2001), 125-147. 
[Lem97] S. Lempp, The computational complexity of torsion-freeness of finitely presented groups, Bull. Austral. Math. Soc. 56 (1997), no. 2, 273-277.

[MS88] J. W. Morgan and P. B. Shalen, Degenerations of hyperbolic structures. III. Actions of 3-manifold groups on trees and Thurston's compactness theorem, Ann. of Math. (2) 127 (1988), no. 3, 457-519.

[MY] I. Mineyev and A. Yaman, Relative hyperbolicity and bounded cohomology, preliminary version, 2006, http://www.math.uiuc.edu/ mineyev/math/.

[Nos93] G. A. Noskov, The T,FA, amenability and continuity properties for groups acting on R-trees, Algebra i Analiz 5 (1993), no. 3, 238-251.

[OP98] K. Ohshika and L. Potyagailo, Self-embeddings of Kleinian groups, Ann. Sci. École Norm. Sup. (4) 31 (1998), no. 3, 329-343.

[Osi] D.V. Osin, Small cancellations over relatively hyperbolic groups and embedding theorems, arXiv:math.GR/0411039.

[Osi06a] D. V. Osin, Relative Dehn functions of amalgamated products and HNN-extensions, Topological and asymptotic aspects of group theory, Contemp. Math., vol. 394, Amer. Math. Soc., 2006, pp. 209-220.

[Osi06b] _ Relatively hyperbolic groups: intrinsic geometry, algebraic properties, and algorithmic problems, Mem. Amer. Math. Soc. 179 (2006), no. 843, vi+100.

[OW] Y. Ollivier and D. T. Wise, Kazhdan groups with infinite outer automorphism group, arXiv:math.GR/0409203.

[Pau88] F. Paulin, Topologie de Gromov équivariante, structures hyperboliques et arbres réels, Invent. Math. 94 (1988), no. 1, 53-80.

[Pau91] - Outer automorphisms of hyperbolic groups and small actions on $\mathbf{R}$-trees, Arboreal group theory (Berkeley, CA, 1988), Math. Sci. Res. Inst. Publ., vol. 19, Springer, 1991, pp. 331-343.

[Rot84] J. J. Rotman, An introduction to the theory of groups, 3rd ed., Allyn and Bacon Inc., Boston, MA, 1984.

[RS94] E. Rips and Z. Sela, Structure and rigidity in hyperbolic groups. I, Geom. Funct. Anal. 4 (1994), no. 3, 337-371.

[Sel95] Z. Sela, The isomorphism problem for hyperbolic groups. I, Ann. of Math. (2) 141 (1995), no. 2, 217-283.

[Sel97] Structure and rigidity in (Gromov) hyperbolic groups and discrete groups in rank 1 Lie groups. II, Geom. Funct. Anal. 7 (1997), no. 3, 561-593.

[Sel99] _ Endomorphisms of hyperbolic groups. I. The Hopf property, Topology 38 (1999), no. 2, 301-321.

[Sel02] Diophantine geometry over groups and the elementary theory of free and hyperbolic groups, Proceedings of the International Congress of Mathematicians, Vol. II (Beijing, 2002), 2002, pp. 87-92.

[Ser77] J.-P. Serre, Arbres, amalgames, SL 2 , Société Mathématique de France, Paris, 1977, Avec un sommaire anglais, Rédigé avec la collaboration de Hyman Bass, Astérisque, No. 46.

[Szc98] A. Szczepański, Relatively hyperbolic groups, Michigan Math. J. 45 (1998), no. 3, 611-618.

[Tuk94] P. Tukia, Convergence groups and Gromov's metric hyperbolic spaces, New Zealand J. Math. 23 (1994), no. 2, 157-187.

[Wat82] Y. Watatani, Property $T$ of Kazhdan implies property FA of Serre, Math. Japon. 27 (1982), no. 1, 97-103.

[Yam04] A. Yaman, A topological characterisation of relatively hyperbolic groups, J. Reine Angew. Math. 566 (2004), 41-89. 
Igor Belegradek, School of Mathematics, Georgia Institute of Technology, AtLANTA, GA 30332-0160

E-mail address: ib@math.gatech.edu

Andrzej Szczepański, Uniwersytet Gdanski, Instytut Matematyki, Wita Stwosza 57, 80-952 Gdansk, POLAND

E-mail address: matas@paula.univ.gda.pl

Oleg V. Belegradek, Department of Mathematics, Istanbul Bilgi University, 80370 Dolapdere-ISTANBUl, TURKEY

E-mail address: olegb@bilgi.edu.tr 